\title{
Gadolinium enhanced MRI in patients with left ventricular apical ballooning syndrome implicates myocarditis as an etiology
}

\author{
Andrew O. Maree ${ }^{1,2^{* \#}}$, Christian Witzke ${ }^{1 \#}$, Godtfred Holmvang ${ }^{1}$, Gregory D. Lewis ${ }^{1}$, Hani Jneid ${ }^{1}$, \\ Lindsay B. Reardon ${ }^{1}$, Nadeem Afridi ${ }^{1}$, Marc J. Semigran ${ }^{1}$, G. William Dec ${ }^{1}$, Harry C. Lowe ${ }^{1}$, \\ Igor F. Palacios ${ }^{1}$ \\ ${ }^{1}$ Cardiology Section, Department of Medicine, Waterford Regional Hospital, Waterford, Ireland \\ ${ }^{2}$ Cardiology Section, Department of Medicine, Massachusetts General Hospital, Boston, USA \\ Email: ${ }^{*}$ andrew.maree@gmail.com
}

Received 2 January 2013; revised 5 February 2013; accepted 15 February 2013

\begin{abstract}
Aims: Left ventricular apical ballooning syndrome (LVABS) is a clinical condition of unknown etiology, characterized by acute onset of atypical apical wall motion and absence of coronary artery disease. Gadolinium-enhanced cardiac magnetic resonance imaging (Gd-MRI) may be used to identify patients with myocarditis. Using cardiac MRI, we evaluated whether acute myocarditis may be an etiology that underlies LVABS. Methods and Results: Consecutive patients who presented with LVABS during a three-year period were included. Demographic data was recorded and echocardiography, coronary angiography, and hemodynamic assessment performed. Gd-MRI was performed in all patients. The study was deemed consistent with myocarditis when global myocardial to skeletal muscle enhancement ratio was $\geq 3.5$. Regional Gd-MRI analysis was also performed. Patients $(n=$ 11) were female $(\mathbf{1 0 0 \% )}$ ) and of mean age 72 years $(72$ \pm 11). Preceding febrile illness occurred in 4 (36.3\%) and leukocytosis in $6(54.4 \%)$ patients. Initial mean left ventricular ejection fraction $(41 \% \pm 12 \%) \mathrm{im}-$ proved $(70.2 \% \pm 8 \%)$ upon follow-up $(39 \pm 43$ days $)$. Global MRI analysis was positive in 5/11 (45.5\%) (mean relative enhancement ratio $4.8 \pm 1.4$ ). Regional MRI analysis was positive in $4 / 6$ further patients (overall: 9/11 (82\%)). Conclusions: Gadolinium enhanced MRI imaging in LVABS implicates myocarditis as a possible etiology. Regional MRI analysis adds sensitivity to global cardiac MRI evaluation.
\end{abstract}

Keywords: Left Ventricular Apical Ballooning Syndrome; Gadolinium-Enhanced Cardiac Magnetic Resonance Imaging; Myocarditis; Catecholamine

*Corresponding author.

${ }^{\#}$ These authors contributed equally to the manuscript.

\section{INTRODUCTION}

Left ventricular apical ballooning syndrome (LVABS) is a clinical condition of unknown etiology that is frequently mistaken for ST-elevation myocardial infarction. Characterized by acute onset of chest pain associated with abnormal LV apical wall motion, the diagnosis requires documentation of patent epicardial coronary arteries by catheterization. Initially described in Japan, isolated cases have been reported in the United States [1-3]. Because this disease typically has an abrupt onset and fast recovery, we hypothesized that acute myocarditis may be an underlying etiology [4-6]. To test this hypothesis, we used Gadolinium enhanced MRI (Gd-MRI) to evaluate 11 consecutive patients who presented to our tertiary care center with findings consistent with left ventricular apical ballooning syndrome.

Myocarditis is defined clinically and pathologically as inflammation of the myocardium. Clinical presentation ranges from non-specific systemic symptoms to fulminant hemodynamic collapse [7]. The diagnosis is generally made based on clinical history and examination, electrocardiograph (ECG) changes (ST segment abnormality, arrhythmias), serological evidence of myocyte necrosis, echocardiography (ECHO), or cinematographic images demonstrating left ventricular dysfunction. In some cases endomyocardial biopsy is performed and may demonstrate cellular infiltration and myocyte degeneration and/or necrosis. Endomyocardial biopsy was previously regarded as the definitive investigation. However, recent studies have highlighted its low sensitivity and inconsistency in its interpretation in the setting of myocarditis, which has led to its decline $[8,9]$.

Decreased performance of endomyocardial biopsy has coincided with the emergence of non-invasive imaging modalities to make the diagnosis of myocarditis. Cardiac magnetic resonance imaging (CMR) has been prominent 
among these modalities and is more frequently being used to diagnose and follow the course of myocarditis $[10,11]$. More recently a systematic review of the use of cardiac MRI to evaluate LVABS described its utility in guiding management [12]. Gadolinium enhanced CMR can detect both early and late signs of myocarditis such as myocardial hyperemia, increased capillary permeability (global (early) relative enhancement) and evidence of myocyte injury (late gadolinium enhancement) [11]. Myocarditis may involve focal areas of myocardium with resultant regional wall motion abnormality [10,11]. Many studies of LVABS have identified an antecedent stressful event that may have triggered the myocardial injury, which appears to result in less myocardial necrosis but a higher level of brain natiuretic peptide than in ST elevation myocardial infarction $[13,14]$. We performed both regional and global analysis of CMR images in consecutive patients presenting with the clinical syndrome of LVABS to identify areas of increased gadolinium uptake consistent with myocardial inflammation.

\section{METHODS}

\subsection{Patient Inclusion and Exclusion Criteria}

The study was conducted in compliance with the Declaration of Helsinki. The study population consisted of 11 consecutive patients who presented to Massachusetts General Hospital with findings consistent with the clinical syndrome of LVAB over a 3-year period. The diagnosis of LVABS was based on strict criteria. All patients presented with acute onset of chest pain, new anterior ECG changes (ST elevation or $\mathrm{T}$ wave inversion) suggestive of an evolving acute myocardial infarction, and elevated cardiac enzymes in the setting of patent coronary arteries upon angiography. Additionally, transient apical wall motion abnormality involving the left ventricular apex and mid-ventricular segments, which extended beyond a single epicardial vascular distribution, was confirmed by ventriculography. The regional wall motion abnormality also had to result in the left ventricle resembling the classically described LVAB syndrome or Takotsubo cardiomyopathy.

Patients with evidence of alternative pathology known to cause acute left ventricular dysfunction were excluded from the study. Intracerebral bleeding, recent cerebral or chest trauma, hypertrophic cardiomyopathy, hypertensive crisis or pheochromocytoma were also criteria for exclusion.

\subsection{Patient Assessment}

Patient demographic details, coronary risk factors, comorbid illnesses, hemodynamic and angiographic findings, laboratory tests, in-hospital events, and follow-up data were recorded and entered into a database.
Cardiac catheterization was performed in all patients within 12 hours of presentation. Left ventriculography and coronary angiography were performed and left ventricular outflow tract systolic gradients measured at rest. Among patients who had no spontaneous gradient, a catheter-induced extrasystolic potentiated beat was used to look for a provokable gradient. A gradient of over 30 $\mathrm{mmHg}$ between the body and the outflow tract of the left ventricle was considered significant.

All patients underwent 2D and Doppler echocardiography at the time of admission, prior to hospital discharge, and upon follow-up. Wall motion abnormalities were identified by 2D echocardiography, and left ventricular ejection fraction was calculated using Simpson's technique. An ejection fraction between $59 \%$ and $75 \%$ was considered normal.

Gadolinium enhanced MRI was performed in all patients using the technique previously described by Friedrich et al. [10] With this technique, both myocardial wall and skeletal muscle signal intensity are measured in T1-weighted spin-echo images acquired in the transverse view of the heart before and immediately following intravenous administration of $0.1 \mathrm{mmol} / \mathrm{Kg}$ Gd-DTPA by hand injection (mean TR: $809 \mathrm{~ms}$, mean TE: $13 \mathrm{~ms}$, matrix size ranging from $256 \times 128$ to $256 \times 192$, mean field of view $240 \mathrm{~mm}$ giving atypical pixel size of approximately $1 \times 1.5-2 \mathrm{~mm}$ ). Mean slice thickness was 7 $\mathrm{mm}$ and 4 signal averages were used. An oblique spatial saturation pulse was used over the atria to reduce flowrelated motion artifacts as well as signal from slowflowing blood in the left ventricle. The myocardial wall and skeletal muscle were segmented manually in preand post-Gadolinium images with cross-referencing between corresponding images. This comparison was done to ensure that regions of interest were well matched in order to sample the same tissue in both sets of images (Figure 1, Panels A and B). Drawing was performed inside the boundaries of the left ventricle to avoid inclusion of signal from epicardial fat or blood pool that could introduce error into the quantitative analysis. Skeletal muscle signal intensity was measured using an elliptical region of interest provided by the MRI software. Signal enhancement for myocardium and skeletal muscle was calculated separately using the following formula: signal intensity post-Gd minus signal intensity pre-Gd divided by signal intensity pre-Gd. Relative myocardial enhancement was calculated by dividing signal enhancement of myocardium by signal enhancement of skeletal muscle. A global relative myocardial enhancement (average of all the analyzable slices through the left ventricle) $\geq 3.5$ was considered consistent with myocarditis.

Freiderich et al. identified focal myocardial enhancement early in the patients' clinical course and confirmed that it subsequently became more diffuse [10]. Therefore, 


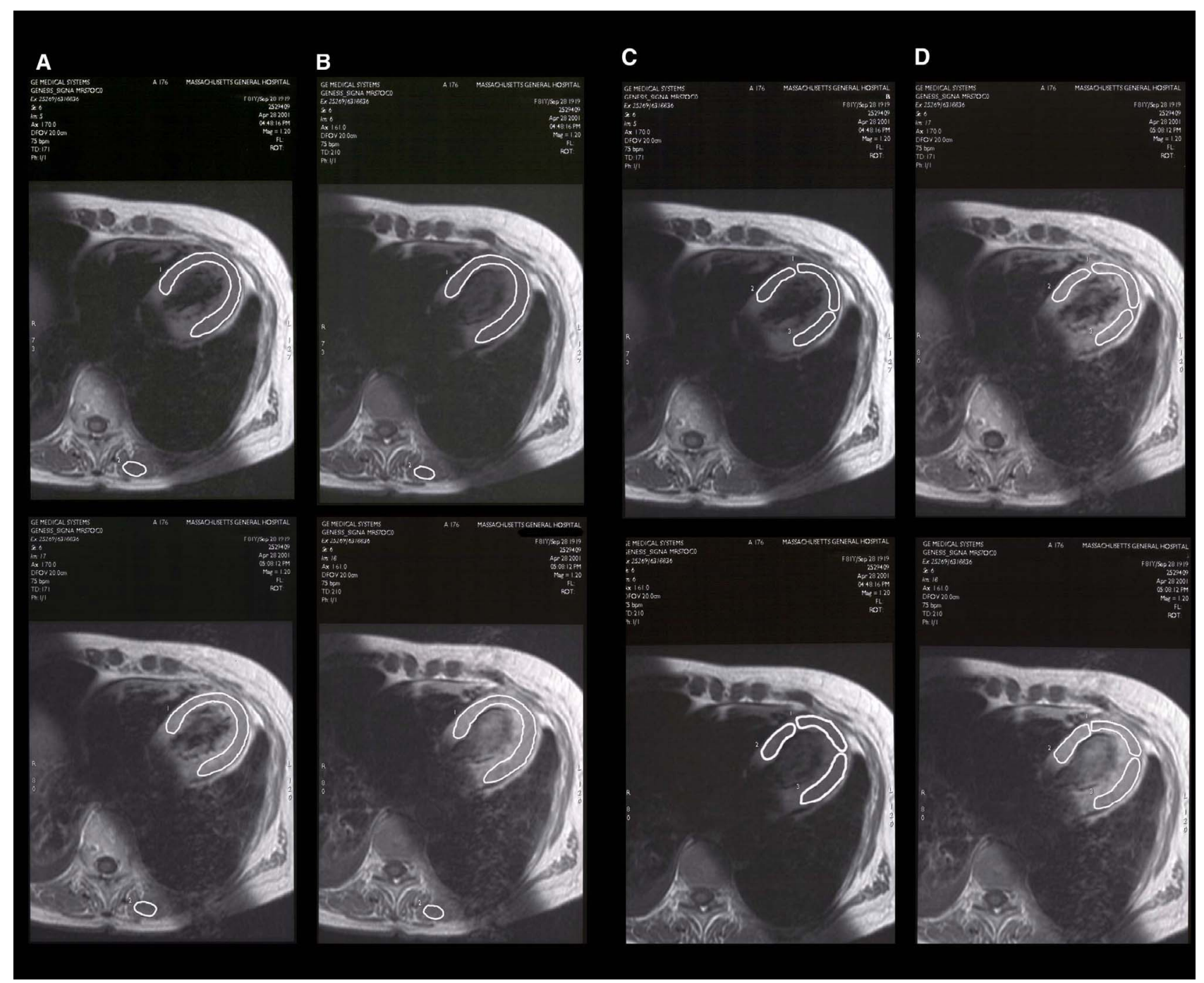

Figure 1. Gadolinium enhanced MRI analysis of the left ventricle in a patient with left ventricular apical ballooning syndrome. This figure shows the similarity between manually drawn segments of the left ventricle chosen before (Panel A) and after (Panel B) injection of Gadolinium for global myocardial analysis. Local drawing that included the septum, apex and posterior wall was performed in the same patient before (Panel C) and after (Panel D) Gadolinium injection in order to calculate the myocardial enhancement index in separate areas. In the image presented, global Gadolinium enhancement analysis (Panels A and B) was negative for Myocarditis (relative myocardial enhancement of 3.4). However, a relative myocardial enhancement of $4.5 \pm 0.9$ was calculated within the apex (wall motion abnormality identified in the ventriculogram) (Panels C and D). This value was greater than the mean value for the normokinetic area $(2.9 \pm 0.6, \mathrm{p} \leq 0.05)$.

in addition to deriving global relative myocardial enhancement for the whole left ventricle, we also calculated local relative myocardial enhancement within the region of the apical wall motion abnormality (area of dyskinetic myocardium) identified by left ventriculography and echocardiography (Figure 1, Panels C and D). This was also referenced to a skeletal muscle region of interest. In this way, patients at different stages in their clinical course and those with focal inflammatory processes might also be detected.

Patient follow-up was carried out $19 \pm 9$ (4 - 27) months after discharge. Follow-up data included survival, NYHA functional class, and whether patients experi- enced recurrent chest pain or re-hospitalization. Followup evaluation was accomplished by direct interview or via the telephone. When necessary, local physicians were contacted for further information or records.

\subsection{Statistical Analysis}

Continuous variables are reported as means \pm standard deviation (SD) and categorical variables as frequencies. Continuous variables were compared by Student $\mathrm{T}$ test, and categorical variables by Fisher's Exact test. A p value $<0.05$ was considered statistically significant. Statistical analysis was performed using the SPSS version 
10.0 statistical package (SPSS Inc., California).

\section{RESULTS}

\subsection{Patient Population}

The study population included 11 consecutive patients presenting to Massachusetts General Hospital between February 1999 and January 2002 with left ventricular apical ballooning syndrome. Baseline demographic and clinical data are shown in Table 1. All patients were female and of mean age $72 \pm 11$ years. All presented with acute onset of chest pain, ST segment and T wave ECG changes, and elevation of cardiac enzymes suggestive of an evolving acute myocardial infarct. ST segment elevation was present in 10 patients and deep T wave inversion in one. In 9 patients the ECG changes were present in the anteroapical and lateral leads, and in 2 patients changes were confined to the lateral leads. One patient with cardiogenic shock required intra-aortic balloon pump support. A preceding febrile illness was present in 4 patients (36\%) and leukocytosis in $6(54 \%)$ patients.

All patients had normal coronary arteries on angiography, and extensive anterior and apical wall motion abnormalities with a hypercontractile left ventricular base on left ventriculography consistent with the classical description of LVAB syndrome (Takotsubo cardiomyopathy) (Figure 2). Spontaneous or provoked dy-

Table 1. Patient population baseline characteristics $(n=11)$.

\begin{tabular}{lc}
\hline DEMOGRAPHIC DATA & $72 \pm 11$ \\
\hline Age (years) & $11(100 \%)$ \\
Female gender & 0 \\
Family history of CAD & $5(45 \%)$ \\
Hypertension & $2(18 \%)$ \\
Smoking & 0 \\
Diabetes Mellitus & 0 \\
Hyperlipidemia & 0 \\
Peripheral vascular disease & 0 \\
Carotid disease & $1(9 \%)$ \\
Chronic Obstructive Airway Disease & \\
CLINICAL CHARACTERISTICS & $11(100 \%)$ \\
Acute onset chest pain & $3(27 \%)$ \\
Preceding febrile viral syndrome & $1(9 \%)$ \\
Preceding stressful situation & $1(9 \%)$ \\
Palpitations & $5(45 \%)$ \\
Dyspnea & $4(36 \%)$ \\
Congestive heart failure & $1(9 \%)$ \\
Cardiogenic shock & $6(54.4 \%)$ \\
Leukocytosis &
\end{tabular}

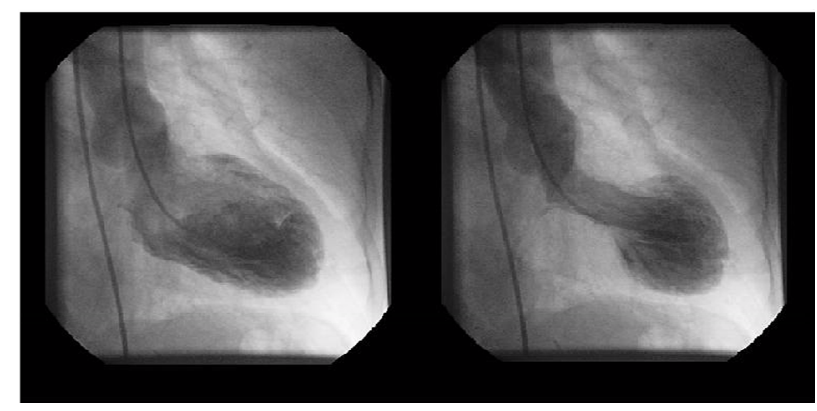

Figure 2. Typical end diastolic (Panel A) and end systolic (Panel B) frames of a left ventriculogram from a patient with apical balloon syndrome exhibiting extensive anterior and apical dyskinesis with a hypercontractile base.

namic left ventricular outflow tract gradient was documented in $4(36 \%)$ patients. Mean initial left ventricular ejection fraction for the whole group was $41 \% \pm 12 \%$.

Seven of eleven patients underwent right heart catheterization. Mean pulmonary artery pressure for this group of patients was $24 \mathrm{mmHg}$ with a pulmonary capillary wedge pressure of $21 \mathrm{mmHg}$.

\subsection{Global Gadolinium Enhanced MRI}

Global myocardial enhancement post Gadolinium relative to skeletal muscle was positive for myocarditis in 5 of 11 patients (45.5\%) (mean value of $4.8 \pm 1.4$ at $5 \pm 2$ days after admission). Of note, Gadolinium enhancement was diffusely distributed throughout the left ventricular wall with scattered patchy areas of higher enhancement.

\subsection{Regional Gadolinium Enhanced MRI}

Use of regional relative myocardial enhancement analysis identified 4 additional patients with changes consistent with myocarditis from the group of patients whose global relative myocardial enhancement did not reach the pre-specified threshold of 3.5 (9/11 overall (82\%)) (Figure 1, Panels $C$ and D). Among these 4 patients, global relative myocardial enhancement was $2.7 \pm 0.5$ (range 2.2 - 3.4), however, relative enhancement within the apical area, where left ventricular wall motion was abnormal, was $4.1 \pm 0.6$ at $2.5 \pm 0.5$ days after admission. This regional value for myocardial enhancement within the dyskinetic segment was significantly greater than the mean value from the remote normokinetic myocardium $(4.1 \pm 0.6$ vs $2.7 \pm 0.4 ; p<0.05)$. Consistent with this, mean relative myocardial enhancement within the apical portion of the left ventricle was also greater than the value associated with normokinetic areas in the 5 patients with global relative myocardial enhancement, indicative of myocarditis $(6.6 \pm 4.3$ vs $3.7 \pm 1.7 ; p<0.05)$. No demographic, clinical, or angiographic characteristics at presentation were identified as predictors of a positive MRI. 


\subsection{Treatment and In-Hospital Events}

All patients required hospital admission (mean stay $8 \pm 3$ days (4 - 13 days)). One patient who presented with cardiogenic shock required intra-aortic balloon pump placement. Dopamine or dobutamine was used in $2(18 \%)$ patients. Two (18\%) patients required intubation. One patient developed atrial fibrillation. There were no inhospital deaths and no other complications were observed.

In-hospital medications included beta-blockers in 9 patients $(82 \%)$, calcium channel blockers in 1 patient (9\%), digoxin in 3 patients (27\%), angiotensin-converting enzyme inhibitors in 8 patients $(73 \%)$, angiotensin receptor blockers in $1(9 \%)$, nitrates in 6 patients $(55 \%)$, and diuretics in 3 patients (27\%). All patients received aspirin and heparin upon presentation and $3(27 \%)$ patients received glycoprotein (GP) IIb/IIIa antagonists. Heparin and GPIIb/IIIa antagonists were stopped after normal coronary arteries were demonstrated by coronary angiography.

All patients improved clinically and echocardiographically. LVEF for the entire group increased from $41 \% \pm$ $12 \%$ to $52 \% \pm 14 \%(\mathrm{p}=0.1)$ at the time of hospital discharge. There was a significant increase of the LVEF at discharge in a sub-group of 9 patients whose initial EF was $\leq 45 \%(37 \pm 5$ to $55 \pm 11, \mathrm{p}=0.01)$. MRI was not a predictor of short or long term outcome.

\subsection{Follow-Up}

Spontaneous recovery of left ventricular ejection fraction to $>50 \%$ was present in all 11 patients at $39.3 \pm 43(1-$ 118 ) days of follow-up (mean LVEF $70.2 \% \pm 7.6 \%$ ). Of note, 3 patients had hyperdynamic left ventricular function one month after presentation. Mean LVEF for this subgroup of patients was $79 \% \pm 2 \%$. At a mean followup period of $18.6 \pm 9.1$ months (median 23 months), all patients were alive, in NYHA functional class I and none had experienced recurrence of chest pain.

\section{DISCUSSION}

The present study provides radiological evidence of myocardial inflammation and implicates myocarditis as an etiology in LVABS. In addition, it suggests that regional analysis of Gadolinium enhanced MR images may identify areas of active inflammation in patients with LVABS. In the early phases of this syndrome, focal myocardial involvement may be the only imaging finding [11].

\subsection{Left Ventricular Apical Ballooning Syndrome}

Left ventricular apical ballooning syndrome is a clinical condition characterized by acute onset of chest pain, electrocardiographic changes (ST segment elevation or depression, abnormal T-waves), elevated cardiac enzymes, and transient left ventricular wall motion abnormality in the presence of angiographically normal coronary arteries. Typically, involvement of the left ventricular apical region is described but more recent reports indicate that the lateral wall, base and right ventricle may also be affected. First described in Japan, recent series describe LVABS in Belgian and American patients [1,2, 15]. In agreement with previous series, elderly females were most frequently diagnosed in our series. Rapid resolution of initially depressed cardiac function has also been previously described [3]. However, hyperdynamic left ventricle function upon follow-up was an unexpected finding.

\subsection{Myocarditis and Left Ventricular Apical Ballooning}

Myocarditis is a pleomorphic disease of infective and non-infective etiology. Patients may be asymptomatic with normal left ventricular systolic function, or have severely compromised left ventricular function and cardiogenic shock [4-7]. Chest pain often occurs and presentation may mimic acute myocardial infarction [16]. LVABS has been strongly associated with catecholamine release and indeed catecholamine induced focal myocarditis and cardiomyopathy are well described [17-20].

A recent report identified a causative viral pathogen in a 67-year-old female with evidence of LVABS and a 3 -week history of flu-like illness. Endomyocardial biopsy and serology confirmed human herpes virus 6 subtype $\mathrm{A}$ as the pathogen, and acute myocarditis was diagnosed by histology and immunohistochemistry [21]. Native heart biopsy is not performed routinely at our institution in line with ACC/AHA recommendations. Therefore, we can not provide histopathological corroboration of our diagnosis of myocarditis.

\subsection{Myocarditis and Cardiac MRI}

At present, endomyocardial biopsy is infrequently performed in patients suspected of having myocarditis. Previously considered the "gold standard", its sensitivity for diagnosing this condition is in doubt and biopsy information rarely influences the management and treatment of these patients [8]. Studies show varying degrees of correlation between histopathology and CMR in myocarditis $[8,9]$.

CMR is being used more frequently to evaluate diverse cardiovascular pathologies with increasing success. It has been used to evaluate infarct size, detect viable myocardium, detect inflammatory change in transplanted hearts, and to diagnose and follow progression of 
myocarditis. CMR has also been used to assess the recovery and guide management of a patients with LVABS [12,22,23].

Friedrich et al. reported their experience with ECG gated, T1-weighted spin-echo images before and after administration of $0.1 \mathrm{mmol} / \mathrm{kg}$ Gadolinium-DTPA in 19 patients with suspected myocarditis [10]. These patients had clinical and laboratory data suggestive of acute myocarditis. This included electrocardiographic changes, reduced systolic function, elevated cardiac enzymes, serological evidence for acute viral infection, exclusion of coronary artery disease, and positive antimyosin scintigraphy. Four of seven patients who underwent endomyocardial biopsy had findings consistent with myocarditis. They determined that a global myocardial enhancement ratio post Gadolinium $\geq 3.5$ relative to skeletal muscle, distinguished patients with myocarditis from normal controls. In a recent case-controlled prospective study of patients with acute myocarditis, CMR had a sensitivity of $76 \%$, specificity of $95.5 \%$, and a diagnostic accuracy of $85 \%$ when early and late gadolinium enhancement and T2-weighted images were combined [11]. In a prior case at our center, myocarditis was diagnosed by gadolinium enhanced MRI using the methods of Freiderich et al. and confirmed by left ventricular biopsy (data not shown). Therefore, in the present study we used gadolinium enhanced MRI as a non-invasive diagnostic tool to detect myocarditis.

Results of global and regional gadolinium-enhanced MRI analysis in the present study suggest that myocarditis is one possible mechanism underlying LVABS. Five patients met the global MRI criterion for myocarditis. When regional analysis was performed in the apical portion of the left ventricle, 4 additional patients were identified with findings consistent with regional myocarditis but did not meet the criteria by global analysis $(9 / 11$ overall $(82 \%))$. We cannot prove histologically that these 4 patients had localized myocarditis, which could account for the initial negative global Gd MRI score. However, presence of extensive regional wall motion abnormality with high local Gd-uptake compared to normally functioning myocardium and absence of coronary artery disease suggest that focal myocardial inflammation was present. Indeed, these findings have been described previously among patients with myocarditis [24]. Furthermore, even in patients whose global relative myocardial enhancement score was consistent with myocarditis, a statistically significant difference for post-Gadolinium enhancement relative to skeletal muscle was found between areas of normal wall motion and the dyskinetic apex. This further supports the hypothesis of differential regional involvement by myocarditis.

Abnormal regional myocardial Gd-uptake has also been described in patients with Hypertrophic Cardio- myopathy [25]. In these patients however, the pattern of myocardial enhancement differs from our group. Absence of asymmetric septal hypertrophy and systolic anterior motion of the anterior leaflet of the mitral valve (by echocardiography) in our patients make this diagnosis unlikely.

\subsection{Stunned Myocardium and Apical Ballooning}

Stunned myocardium due to transient microvascular vasospasm, microvascular thrombosis with spontaneous lysis, and direct myocardial injury has been proposed as a cause of transient left ventricular dyskinesis. However, two reports of myocardial biopsy in the setting of transient left ventricular dyskinesis resembling LVABS have been published. In both, the authors found focal myocyte injury which is not consistent with the concept of stunning $[21,26]$. Transient ischemic episodes due to epicardial coronary artery occlusion from spasm or thrombosis may lead to stunned myocardium or reperfusion injury. Regional cardiac MRI signal enhancement post gadolinium and wall motion abnormalities have been reported in this setting [27]. However, in our study anteroapical, lateral, and inferior ventricular wall dysfunction coexisted with normal coronary arteries. Even transient proximal left anterior descending coronary artery occlusion is unlikely to account for such extensive wall motion abnormalities.

\subsection{Catecholamine Myocarditis and Cardiomyopathy}

Myocardial dysfunction mediated by catecholamine cardiotoxicity is well described and found most frequently in the context of catecholamine-secreting tumors. Indeed a focal pattern of myocarditis has been described in a patient with phaeochromocytoma [20]. Catecholamine myocarditis has been detected in patients abusing beta agonists, and implicated as a mechanism in patients in whom cardiomyopathy and panic disorder coincide [28, 29]. Others have also associated LVABS and catecholamine surges and LVABS post dobutamine stress echo has been described $[17,30]$. In one recently published series, the authors elicited a history of profound psychological or physical stress in the 24 hours preceding onset of chest pain in each of their patients with LVABS [3]. Others recorded elevated norepinephrine concentrations in $74 \%$ of patients in their cohort [31]. To date most LVABS studies have identified a strong female preponderance to the condition as in our study. This may reflect a particular vulnerability of elderly post-menopausal female patients to catecholamine surges that induce ventricular dysfunction [3]. Indeed in animal models oestrogen has been shown to attenuate this response [32,33]. 
Abnormal coronary microcirculation has been postulated as the etiology of LVABS, however, studies show mixed results $[34,35]$. An abnormal microcirculation pattern determined by response to intracoronary acetylcholine has been reported in patients with diffuse left ventricular dysfunction and those suspected of having myocarditis [36]. Thus, this abnormal response may represent a unifying mechanism for myocarditis and microvascular disease in LVABS. However, it remains unsubstantiated [37]. One possibility is that LVABS emerges when vulnerable myocardial substrate is exposed to a significant stressor such as transient elevation in catecholamine levels.

In conclusion, as with many syndromes it is unlikely that one pathological process explains all cases of LVABS. A composite of several processes is likely to be involved. However, the clinical course, area of myocardial involvement, absence of coronary obstruction, rapid improvement in LV function, and positive gadolinium enhanced MRI implicate an inflammatory myocardial etiology.

\section{Study Limitations}

Our study is limited by the relatively small number of patients and lack of histological data to correlate with CMR findings. LVABS is however a relatively rare condition that is only now increasingly appreciated. Routine myocardial biopsy is less frequently performed due to inconsistency of the histological findings and interpretation and invasive nature of the biopsy procedure. In many cases myocardial biopsy has been replaced my CMR imaging. Our study is however a prospective analysis of consecutive patients and provides additional understanding of the etiology that may underlie LVABS.

\section{REFERENCES}

[1] Dote, K., Sato, H., Tateishi, H., Uchida, T. and Ishihara, M. (1991) Myocardial stunning due to simultaneous multivessel coronary spasms: A review of 5 cases. Journal of Cardiology, 21, 203-214.

[2] Villareal, R.P., Achari, A., Wilansky, S. and Wilson, J.M. (2001) Anteroapical stunning and left ventricular outflow tract obstruction. Mayo Clinic Proceedings, 76, 79-83. doi:10.4065/76.1.79

[3] Sharkey, S.W., Lesser, J.R., Zenovich, A.G., Maron, M.S., Lindberg, J., Longe, T.F. and Maron, B.J. (2005) Acute and reversible cardiomyopathy provoked by stress in women from the United States. Circulation, 111, 472479. doi:10.1161/01.CIR.0000153801.51470.EB

[4] Johnson, R.A. and Palacios, I. (1982) Dilated cardiomyopathies of the adult (first of two parts). The New England Journal of Medicine, 307, 1051-1058. doi:10.1056/NEJM198210213071704

[5] Johnson, R.A. and Palacios, I. (1982) Dilated cardiomyo- pathies of the adult (second of two parts). The New England Journal of Medicine, 307, 1119-1126.

doi:10.1056/NEJM198210283071804

[6] Dec, G.W., Jr., Palacios, I.F., Fallon, J.T., Aretz, H.T., Mills, J., Lee, D.C. and Johnson, R.A. (1985) Active myocarditis in the spectrum of acute dilated cardiomyopathies. Clinical features, histologic correlates, and clinical outcome. The New England Journal of Medicine, 312, 885-890. doi:10.1056/NEJM198504043121404

[7] Magnani, J.W. and Dec, G.W. (2006) Myocarditis: Current trends in diagnosis and treatment. Circulation, 113, 876-890. doi:10.1161/CIRCULATIONAHA.105.584532

[8] Mason, J.W., O’Connell, J.B., Herskowitz, A., Rose, N.R., McManus, B.M., Billingham, M.E., Moon, T.E. and the Myocarditis Treatment Trial Investigators (1995) A clinical trial of immunosuppressive therapy for myocarditis. The New England Journal of Medicine, 333 269-275. doi:10.1056/NEJM199508033330501

[9] Chow, L.H., Radio, S.J., Sears, T.D. and McManus, B.M. (1989) Insensitivity of right ventricular endomyocardial biopsy in the diagnosis of myocarditis. Journal of the American College of Cardiology, 14, 915-920. doi:10.1016/0735-1097(89)90465-8

[10] Friedrich, M.G., Strohm, O., Schulz-Menger, J., Marciniak, H., Luft, F.C. and Dietz, R. (1998) Contrast media-enhanced magnetic resonance imaging visualizes myocardial changes in the course of viral myocarditis. Circulation, 97, 1802-1809. doi:10.1161/01.CIR.97.18.1802

[11] Abdel-Aty, H., Boye, P., Zagrosek, A., Wassmuth, R., Kumar, A., Messroghli, D., Bock, P., Dietz, R., Friedrich, M.G. and Schulz-Menger, J. (2005) Diagnostic performance of cardiovascular magnetic resonance in patients with suspected acute myocarditis: Comparison of different approaches. Journal of the American College of Cardiology, 45, 1815-1822. doi:10.1016/j.jacc.2004.11.069

[12] Leurent, G., Larralde, A., Boulmier, D., Fougerou, C., Langella, B., Ollivier, R., Bedossa, M. and Le Breton, H. (2009) Cardiac MRI studies of transient left ventricular apical ballooning syndrome (takotsubo cardiomyopathy) A systematic review. International Journal of Cardiology, 135, 146-149. doi:10.1016/j.ijcard.2009.03.067

[13] Prasad, A., Madhavan, M. and Chareonthaitawee, P. (2009) Cardiac sympathetic activity in stress-induced (Takotsubo) cardiomyopathy. Nature Reviews Cardiology, 6, 430-434. doi:10.1038/nrcardio.2009.51

[14] Madhavan, M., Borlaug, B.A., Lerman, A., Rihal, C.S. and Prasad, A. (2009) Stress hormone and circulating biomarker profile of apical ballooning syndrome (Takotsubo cardiomyopathy): Insights into the clinical significance of B-type natriuretic peptide and troponin levels. Heart, 95, 1436-1441. doi:10.1136/hrt.2009.170399

[15] Desmet, W.J., Adriaenssens, B.F. and Dens, J.A. (2003) Apical ballooning of the left ventricle: First series in white patients. Heart, 89, 1027-1031. doi:10.1136/heart.89.9.1027

[16] Dec, G.W., Jr., Waldman, H., Southern, J., Fallon, J.T., Hutter, A.M., Jr. and Palacios, I. (1992) Viral myocarditis mimicking acute myocardial infarction. Journal of the 
American College of Cardiology, 20, 85-89. doi:10.1016/0735-1097(92)90141-9

[17] Margey, R., Diamond, P., McCann, H. and Sugrue, D. (2009) Dobutamine stress echo-induced apical ballooning (Takotsubo) syndrome. European Journal of Echocardiography, 10, 395- 399. doi:10.1093/ejechocard/jen292

[18] Kassim, T.A., Clarke, D.D., Mai, V.Q., Clyde, P.W. and Mohamed Shakir, K.M. (2008) Catecholamine-induced cardiomyopathy. Endocrine Practice, 14, 1137-1149.

[19] Imperato-McGinley, J., Gautier, T., Ehlers, K., Zullo, M.A., Goldstein, D.S. and Vaughan Jr., E.D., (1987) Reversibility of catecholamine-induced dilated cardiomyopathy in a child with a pheochromocytoma. The New England Journal of Medicine, 316, 793-797. doi:10.1056/NEJM198703263161307

[20] Van Vliet, P.D., Burchell, H.B. and Titus, J.L. (1966) Focal myocarditis associated with pheochromocytoma. The New England Journal of Medicine, 274, 1102-1108. doi:10.1056/NEJM196605192742002

[21] Bigalke, B., Klingel, K., May, A.E., Beyer, M., Hovelborn, T., Kandolf, R. and Gawaz, M. (2005) Serious clinical course of myocarditis with "apical ballooning": First presentation of pathogenicity of HHV6 subtype A in myocarditis. Internist (Berlin), 46, 1259-1264. doi:10.1007/s00108-005-1494-6

[22] Demaria, A.N., Ben-Yehuda, O., Berman, D., Feld, G.K., Ginsberg, J., Greenberg, B.H., Lew, W.Y., Sahn, D. and Tsimikas, S. (2006) Highlights of the year in JACC 2005. Journal of the American College of Cardiology, 47, 184302. doi:10.1016/j.jacc.2005.11.020

[23] Balaguer, J.R., Estornell, J., Planas, A.M., Jacas, V. and Nadal, M. (2005) Transient left ventricular apical ballooning and cardiac magnetic resonance. International Journal of Cardiology, 135, 146-149.

[24] Sarda, L., Colin, P., Boccara, F., Daou, D., Lebtahi, R., Faraggi, M., Nguyen, C., Cohen, A., Slama, M.S., Steg, P.G. and Le Guludec, D. (2001) Myocarditis in patients with clinical presentation of myocardial infarction and normal coronary angiograms. Journal of the American College of Cardiology, 37, 786-792. doi:10.1016/S0735-1097(00)01201-8

[25] Wilson, J.M., Villareal, R.P., Hariharan, R., Massumi, A., Muthupillai, R. and Flamm, S.D. (2002) Magnetic resonance imaging of myocardial fibrosis in hypertrophic cardiomyopathy. Texas Heart Institute Journal, 29, 176-180.

[26] Kawai, S., Suzuki, H., Yamaguchi, H., Tanaka, K., Sawada, H., Aizawa, T., Watanabe, M., Tamura, T., Umawatari, K., Kawata, M., Nakamura, T., Yamanaka, O. and Okada, R. (2000) Ampulla cardiomyopathy ("Takotusbo" cardiomyopathy) - Reversible left ventricular dysfunction: With ST segment elevation. Japanese Circulation Journal, 64, 156-159. doi:10.1253/jcj.64.156

[27] McNamara, M.T., Higgins, C.B., Ehman, R.L., Revel, D., Sievers, R. and Brasch, R.C. (1984) Acute myocardial ischemia: Magnetic resonance contrast enhancement with gadolinium-DTPA. Radiology, 153, 157-163.
[28] Nino, A.F., Berman, M.M., Gluck, E.H., Conway, M.M., Fisher, J.P., Dougherty, J.E. and Rossi, M.A. (1987) Drug-induced left ventricular failure in patients with pulmonary disease. Endomyocardial biopsy demonstration of catecholamine myocarditis. Chest, 92, 732-736. doi:10.1378/chest.92.4.732

[29] Kahn, J.P., Drusin, R.E. and Klein, D.F. (1987) Idiopathic cardiomyopathy and panic disorder: Clinical association in cardiac transplant candidates. American Journal of Psychiatry, 144, 1327-1330.

[30] Wittstein, I.S., Thiemann, D.R., Lima, J.A., Baughman, K.L., Schulman, S.P., Gerstenblith, G., Wu, K.C., Rade, J.J., Bivalacqua, T.J. and Champion, H.C. (2005) Neurohumoral features of myocardial stunning due to sudden emotional stress. The New England Journal of Medicine, 352, 539-548. doi:10.1056/NEJMoa043046

[31] Gianni, M., Dentali, F., Grandi, A.M., Sumner, G., Hiralal, R. and Lonn, E. (2006) Apical ballooning syndrome or takotsubo cardiomyopathy: A systematic review. European Heart Journal, 27, 1523-1529. doi:10.1093/eurheartj/ehl032

[32] Ueyama, T., Hano, T., Kasamatsu, K., Yamamoto, K., Tsuruo, Y. and Nishio, I. (2003) Estrogen attenuates the emotional stress-induced cardiac responses in the animal model of Tako-tsubo (Ampulla) cardiomyopathy. Journal of Cardiovascular Pharmacology, 42, S117-119. doi:10.1097/00005344-200312001-00024

[33] Fukui, M., Mori, Y., Tsujimoto, S., Takehana, K., Sakamoto, N., Kishimoto, N., Imada, T., Maeba, H., Nose, A., Yamahara, H., Kijima, Y., Kitamura, T., Ueyama, T., Kikuchi, S., Tokoro, T., Masaki, H., Nishikawa, M. and Iwasaka, T. (2006) "Takotsubo" cardiomyopathy in a maintenance hemodialysis patient. Therapeutic Apheresis and Dialysis, 10, 94-100. doi:10.1111/j.1744-9987.2006.00308.x

[34] Ako, J., Takenaka, K., Uno, K., Nakamura, F., Shoji, T., Iijima, K., Ohike, Y., Kim, S., Watanabe, T., Yoshizumi, M. and Ouchi, Y. (2001) Reversible left ventricular systolic dysfunction-Reversibility of coronary microvascular abnormality. Japanese Circulation Journal, 42, 355363. doi:10.1536/jhj.42.355

[35] Abe, Y., Kondo, M., Matsuoka, R., Araki, M., Dohyama, K. and Tanio, H. (2003) Assessment of clinical features in transient left ventricular apical ballooning. Journal of the American College of Cardiology, 41, 737-742. doi:10.1016/S0735-1097(02)02925-X

[36] Mathier, M.A., Rose, G.A., Fifer, M.A., Miyamoto, M.I., Dinsmore, R.E., Castano, H.H., Dec, G.W., Palacios, I.F. and Semigran, M.J. (1998) Coronary endothelial dysfunction in patients with acute-onset idiopathic dilated cardiomyopathy. Journal of the American College of Cardiology, 32, 216-224. doi:10.1016/S0735-1097(98)00209-5

[37] Dec, G.W. (2005) Recognition of the apical ballooning syndrome in the United States. Circulation, 111, 388-390. doi:10.1161/01.CIR.0000155234.69439.E4 